\title{
Exploring Semantic Relationships Across Internet Resources
}

\author{
H. Castro, M. T. Andrade, F. Almeida \\ Fac. de Eng. Universidade Porto, INESC Porto \\ Porto, Portugal \\ Leonardo Chiariglione \\ CEO, CEDEO.net \\ Via Borgionera, 103 - I-10040 Villar Dora (TO) Italy
}

\author{
G. Tropea, N. Blefari Melazzi \\ UdR Roma Tor Vergata \\ CNIT \\ Rome, Italy \\ Aziz S. Mousas, Dimitra I. Kaklamani \\ School of Electrical and Computer Engineering \\ NTUA \\ Athens, Greece
}

\begin{abstract}
As the social character of the Web grows stronger, online multimedia resources become more tangled, exhibiting multiple and complex relationships. Often, these relationships are not easily spotted by search engines because they are not explicit. Still, when searching for content, users would certainly appreciate to receive results indicating all resources that are somehow related to their topic of interest without having to explicitly request for it. In spite of this trend, current technology for declaring and describing resources on the Internet as well as their inter-relationships is very much fragmented and the most commonly used standards, such as HTML, do not offer adequate functionality. While the goal of the MPEG-21 standard is to provide an all encompassing, universal and standard way to perform the declaration of digital resources, the work being developed in the IST CONVERGENCE project has shown that this standard, (specifically its part 3, Digital Item Identification), also has a limited ability to specify relationships between digital resources. This document presents a proposed extension to MPEG-21 Digital Item Identification, which enables it to support a uniform, semantically expressive and precise specification of the relationships between MPEG-21 digital items, rendering it a much more effective tool for a ubiquitous representation and interconnection of digital resources.
\end{abstract}

Keywords-component; MPEG-21, DID, DII, VDI, Semantic Web, Content Centric Internet, Metadata, RDF/OWL

\section{INTRODUCTION}

The Internet was originally conceived as a community of hosts that cooperate to support the exchange of unstructured information between its nodes. Today, in contrast, it is becoming a collective of things, people, and, especially, information items and services [1]. This system is evolving towards a "content-centric" paradigm [2], where, the key elements are no longer the "hosts", but instead, the exchanged data and services (or content).

This shift has spurred new forms of declaring information objects and new types of interoperations between web based information services. However, current technology and practices enable only the expression and establishment of very rigid and semantically mute or ambiguous connections among on-line resources.
For instance, the only standard and universal way of declaring a connection between two digital documents is to insert in one of them the URL of the other. This is typically done in HTML through the employment of the $\langle a\rangle$ or $<$ link $>$ tags, which indicate that the referenced document is somehow related to the referencing one. These tags' specification [3] includes some support for the expression of the nature/type of the declared relationships but the expressible relationship types are very few, and still semantically ambiguous. Furthermore, most, if not all, of the major on-line content retrieval and access systems (browsers) make no use of this information.

The present Internet scenario is thus characterized by a semantically poor and fractured expression and manipulation of logical interconnections among digital resources. It is our belief that the availability of standardized mechanisms that exploit the interrelations of on-line resources can be a significant driver to the fulfillment of the above-referred content-centric paradigm. On one side it will allow a more efficient distribution of media resources on the Internet, based on their semantic content and relationships, subsequently enhancing the performance of content-based search engines. On the other side, it will contribute to the seamless access to resources, thus increasing the degree of satisfaction of users as well as business opportunities.

This paper describes work conducted within the European-funded FP7-ICT CONVERGENCE [4] project, to identify requirements within this context and design an adequate solution for declaring and using semantic relationships among on-line resources. It is structured in the following way:

- Section II contextualizes the CONVERGENCE project, by presenting its motivation, its key concepts and exploiting some of its use cases to demonstrate the need for inter-Digital resources' relationships expression mechanisms.

- Section III exposes MPEG-21's limitation for the expression of said needs.

- Section IV presents the proposed mechanism to enable MPEG-21 to explicitly and efficiently declare relationships between digital resources. 
- Section V presents some exploitation possibilities of the proposed mechanism.

- Section VI contains the final conclusions.

\section{CONVERGENCE PROJECT}

\section{A. Motivation}

A key component of the "content-centric" paradigm is content-centric networking: the ability of the network layer to provide users with contents, instead of providing communication channels between hosts, and to be aware of such contents, at least in the sense of knowing the "name" of the contents [9]. However, such an evolved network layer needs to be complemented by corresponding contentcentered solutions at higher layers also, and by the way contents themselves are organized, structured and used. As a matter of fact, the content-centric operational paradigm imposes new requirements on the data manipulating provisions that make up the Internet. Current solutions addressing these issues are proprietary, non-interoperable, restricted to specific classes of information, and lacking in key functionality required by providers and consumers of digital information.

One example is Google's "related:" search option, which is supposed to find pages related to another specific page, but often blatantly fails when conceptual similarity is expected by the user (example: "related: ieeexplore.ieee.org").

The most successful Internet initiatives that cover key areas of human behavior, like social portals as YouTube or Facebook, repositories of scientific knowledge like IEEEXplore and all online shops, have, as the key of their success, the exploitation they perform, of the knowledge that some content is related (in human understandable terms) to some other content they offer (the "I like it" button, scientific papers citing other papers, goods linked to other goods because other people have bought similar ones). Such linking techniques exploit, in the best cases, the tags that are given by users to the content they publish, or try to parse and aggregate based on textual comments.

We argue that, given the right means, users are ready to refine the tags they pin on content, by exploiting more sophisticated semantic models and the possibility to declare semantic relations between specific, self-contained, digital objects, effectively bridging them to build a web of semantically connected digital items.

In order to persuade and lead them to spend the extra effort needed to perform a semantically-rich linking of their content to other content, it is crucial that a widely supported standard for the definition of such links be approved and introduced. Furthermore, easy-to-use tools exploiting the proposed standard are needed, as well as simple and shared ontology models that organize, in clear and extensible ways, the taxonomy of possible relationships.

Within the outlined context, the strategic goal of the CONVERGENCE project is to address these new demands, through a novel framework supported by a content-based, publish-subscribe service model to complement and enhance the current Internet architecture.

\section{B. MPEG-21 and VDI Concept}

One of the key enablers of today's media revolution has been the emergence of broadly accepted standards (e.g. HTML, XHTML, PDF, etc). Such standards are centered on the "classical" needs of information representation and exchange, that were typical of an age were the roles of information producers and consumers were clearly established and different. Today, however, this once clear distinction is getting increasingly blurred as the requirements of digital information users and producers are effectively converging.

MPEG standards are amongst those that most broadly address these needs. MPEG-2 1 already defines standard ways of providing meta-information to describe the content and structure of complex media resources designated as "Digital Items" (DIs) [5]. It provides the means to universally declare, exchange, manage and trade digital media objects. This has been found synergistic with the CONVERGENCE project idea of extending this ability to a broader range of resources, including real world objects, services and people. To accomplish this goal, CONVERGENCE employs and extends the MPEG-21 standard for the declaration of any digital object, defining the Versatile Digital Item (VDI).

The VDI is thus acting as the fundamental information unit for transaction and distribution in the CONVERGENCE framework. A VDI is a general purpose container of information about real world objects, services, people and digital resources. VDIs, therefore, bind meta-information (describing the content and structure of the item) and resources (other VDIs, audio, images, video, text, html, etc.).

However, if VDIs are to rely solely on already standardized MPEG-21 mechanisms, they won't be able to provide the functionality needed to overcome the referred limitations and take full advantage of semantic relationships across resources in the Internet. The remainder of this section presents a set of use cases developed within the CONVERGENCE project that highlight the benefits that can be gained when exploiting these semantic relationships.

\section{Use Cases}

Two representative, although simple and very specific, CONVERGENCE use cases are briefly described in this section. They refer to different applications areas, exploiting the VDI features while exposing the need for tools to perform the declaration of inter-VDI relationships.

1) Collaborative Content Enrichment: an important part of Simon's audiovisual documentation on Occitan culture is, naturally, in Occitan. This can be a barrier for people who want to use the material for teaching. To try to overcome this problem, Simon decides to make available, on-line, part of this documentation, allowing and expecting that interested bilingual users will produce a translation. He thus selects a part of his audiovisual documentation and produces a corresponding VDI including the necessary licenses for other people to use it. Carla and Jim download the VDI and use it to produce their own partial translations into Italian and English. Upon 
Simon's acceptance of their translations, the corresponding VDIs are published on Simon's video site.

The new VDIs created by Carla and Jim have a clear logical relationship with Simon's original VDI. The explicit and unambiguous declaration of that relationship in terms of an "enrichment" of the original VDI, would enable the automatic linking between the VDIs. Consequently, regardless of their physical location, users would be able to "browse" between such VDIs. The possibility for users to seamlessly and automatically obtain the translation VDI while performing a search for Simon's audiovisual Occitan documentation, would certainly be an added-value both for the users as well as for Simon, who would see his potential audience increasing.

2) Augmented e-Learning: Professor Watson publishes a VDI containing the material she lectures in each class, as a lecturing episode. Each such VDI is made available for subscription to all students. Tom, a student who subscribed to the course, is automatically notified about the release of new lecture episodes. While watching the latest episode, he comes up with a doubt about a lectured subject. He produces a new VDI with a comment pointing to the subject he has doubts about, and makes it public. The CONVERGENCE system then pushes the comment VDI to all subscribed students. They start a group discussion and work out a solution for Tom's problem.

Tom's VDI presents a "commenting" relationship with the lecture VDI. The existence of a VDI mechanism to explicitly declare this relationship, would allow the system to automatically distribute Tom's comment to all users who subscribed the lectures' VDIs, without requiring any additional action from their side. This feature allows creating much richer and engaging e-Learning scenarios, with clear benefits for the participants.

\section{The Need for Relationships}

The use cases presented above demonstrate that there are effectively a number of inter-relations between resources that can provide significant added-value, if thoroughly exploited. They thus highlight the need for tools to allow the expression, of the relationships between information units, i.e., the VDIs within the CONVERGENCE eco-system, in a semantically rich and precise way. Such a tool would enable the weaving of a semantically unambiguous interconnecting web, which traverses all of CONVERGENCE's information objects, and together with them, forms an automatically navigable informational superstructure.

\section{MPEG-21 SHORTCOMMINGS}

As referred in section II, MPEG-21 already brings additional value to the multimedia delivery chain by being able to integrate diverse technologies and providing a coherent and open representation for media resources. The MPEG-21 Digital Item Declaration (DID) standard [6] provides the tools for declaring the structure of DIs, the parts that compose it and how they interrelate. MPEG-21 Digital
Item Identification (DII) standard [8], provides the tools to (among other things), uniquely identify Digital Items and link Digital Items with related information.

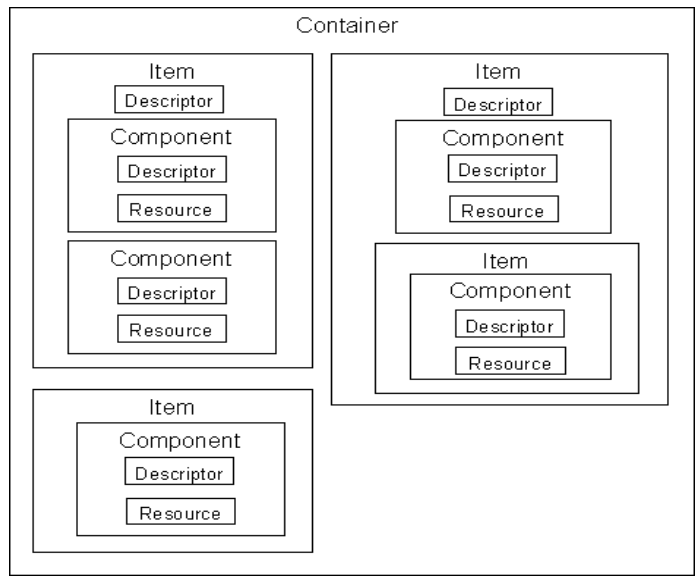

Figure 1 - MPEG-21 DI Declaration Example

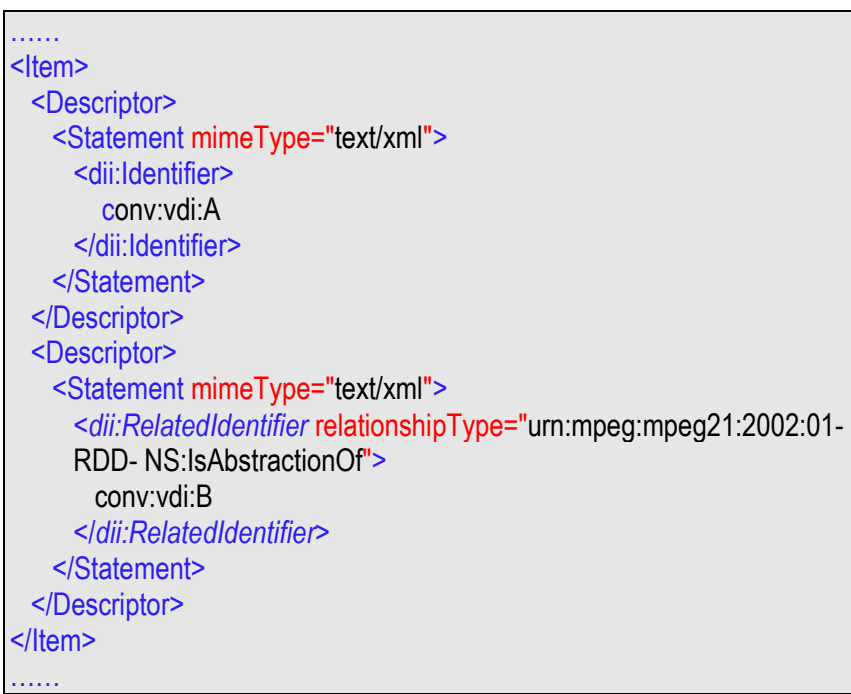

Figure 2 - MPEG-21 DI Declaring a Relationship

However, both these tools perform only a rigid, limited and, generally, implicit description of inter Digital Item relationships. MPEG-21 basically enables: (through DID) the implicit expression of (only), containment/composition relationships between DIs (present within the same declaration), through the placing of (XML) elements within elements according to a strict linear hierarchy. This is depicted in Figure 1; (through DII) the explicit expression of a limited set of inter-DI relationships, through the employment of dii:RelatedIdentifier elements and their relationshipType attribute, as presented in Figure 2.

The earlier scheme does not allow the declaration of relationships other than those of "containment/composition", which are in effect at the time of DI declaration redaction. The later scheme is more versatile and enables an explicit declaration of a greater variety of inter-DI relationships. 
Nonetheless, it is still insufficient as a fully fledged tool for the expression of said relationships.

The dii:RelatedIdentifier element was semantically empowered through an amendment [7] to MPEG-21 Part 3 [8], that enriched that element with the capability to express the nature of the relationship. Still the available pool of relationship types is very small, and though it is extendible, such an extension requires the registration of the new type with the Registration Authority for ISO/IEC. This is an unpractical scheme for the definition of relationship taxonomies. It is also completely divorced from the $\mathrm{RDF} / \mathrm{OWL}$ universe, which would be the natural venue for the expression of the logical connections in scope.

In light of the above, the tools defined in [7], are immature. Their employment for the webbing of the DI logical inter-relational tissue would lead to contrived metadata constructs that would be hard or impossible to query through common and widespread technologies such as SPARQL. Furthermore, said tools do not allow the expression of relationships of higher complexity such as non-binary relationships, or property endowed relationships. For instance, the declaration of a relationship between $D I A$ and $D I B$, which has a time instant as its property, is not expressible when relating DIs through dii:RelatedIdentifier. If an RDF/OWL based approach is employed, the solution becomes trivial as the relationship is simply modeled as "relational" class that maintains a "hasSubject" relationship with $D I A$, a "hasObject" relationship with $D I B$ and a "hasTime" relationship with said time instant.

In summary, the standard does not allow for a versatile expression of non-hierarchical and complex inter-DI relationships, which indeed occur in reality, as highlighted in the CONVERGENCE's use cases. Moreover, the declared relationship is always materialized in the form of a URI/URL to provide the physical location of the component. This scheme is very much oriented towards the original concepts of the Internet and not towards the new content-centric paradigm, where each resource should be referred via its name and semantic attributes.

The possibility to explicitly declare complex inter-DI semantic relationships within MPEG-21 DII, would be a simple and powerful way to establish a rich semantic interwebbing of the global DI tissue. In our socially interconnected digital world, this would greatly empower application and systems which employ MPEG-21, lending them a greater social usefulness and overall value for the users.

In the remainder of this article a mechanism that supports such a declaration is specified and some of its immediate potential uses, within CONVERGENCE, are explored.

\section{DI RELATING MECHANISM}

In order to be in accordance with MPEG-21 DID's "culture", the declaration of inter-DI relationships should be performed through the introduction of inter-DI relationship declaring did:Descriptors. Each such did:Descriptor binds, to its parent did:Item element (which represents a DI), a portion of DII metadata.

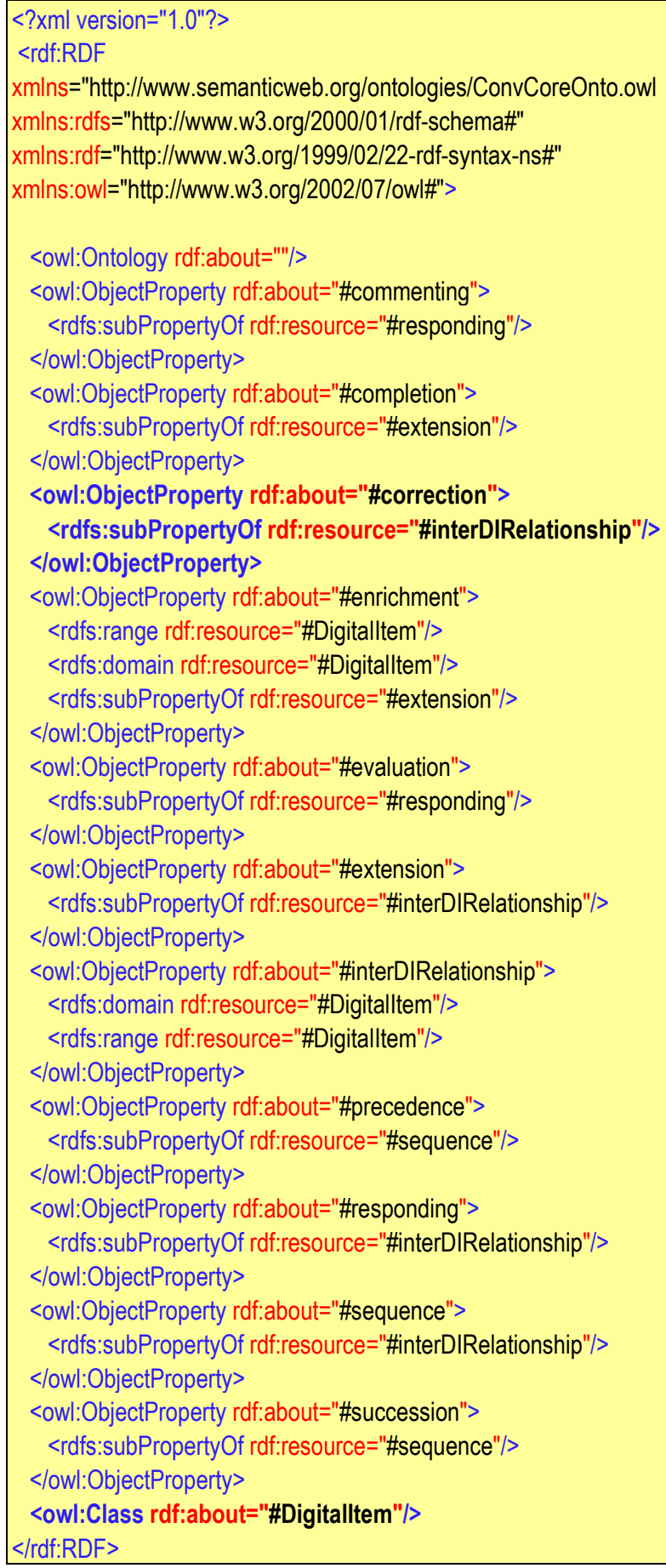

Figure 3 - Inter-DI Relationship OWL Ontology

This DII metadata should be a newly added DII element, named dii:Relationships, (that we are proposing), that replaces the current dii:RelatedIdentifier. This new DII element should carry an RDF/OWL metadata payload, which describes the relationships in which the described DI 
participates with other DIs. These relationships are expressed as RDF/OWL subject-predicate-object triples. In each such triple a DI may be either the subject or the object. The predicate denotes the relationship between them and is a property of an inter-DI ontology.

For instance, if $D I A$ is a correction to $D I B$, then, it may be said that $D I A$ "corrects" $D I B$, that is, $D I A$ is logically bound to $D I B$ through the relationship of "correction". In this example $D I A$ is the subject, $D I B$ the object and correction is the predicate.

In accordance with the previous example, Figure 3 presents a possible base Inter-DI relationship classification ontology (which also is graphically depicted in Figure 4). In it, relationships (such as correction), are modeled as owl:ObjectProperty, and entities (such as Digital Item), are modeled as owl:Class.

The universe of expressible inter-DI relationships should be defined in a global ontology, which may, in its turn, be easily extended by local or domain ontologies.

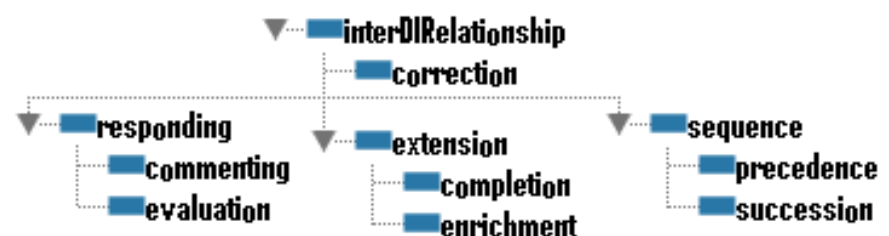

Figure 4 - Relationship Ontology Depiction

The mechanism here presented, is potentially universal, as it relies on open standards, and provides a contribution and a practical solution to the establishment of an efficient content-centric Internet.

\section{MECHANISM EXPLOITATION}

The exploitation of the previously defined mechanism in the declaration/publication, retrieval and consumption of a DI implies two fundamental steps:

- Initially, the DI's (or VDI's, in CONVERGENCE's specific case) declaration is produced. It carries metadata describing the declared DI and its relationships to other DIs. The DI declaration is thus "inscribed" into the overall web of DI declarations and may then be disseminated;

- At the time of DI consumption, search, automatic manipulation, etc., the relating metadata should be analyzed in order to apprehend the relationships between DIs and thus enrich the interaction with them.

The following subsections present two examples of how the defined, relational empowering, extension to MPEG-21 DII, is employed and exploited within the context of CONVERGENCE and may be done so elsewhere.

\section{A. DI Relationship Declaration}

Figure 5 exemplifies the simplicity with which this mechanism enables an MPEG-21 DI declaration to specify that the declared DI (conv:di:005), maintains a relationship of "enrichment", towards another DI (conv:di:006).

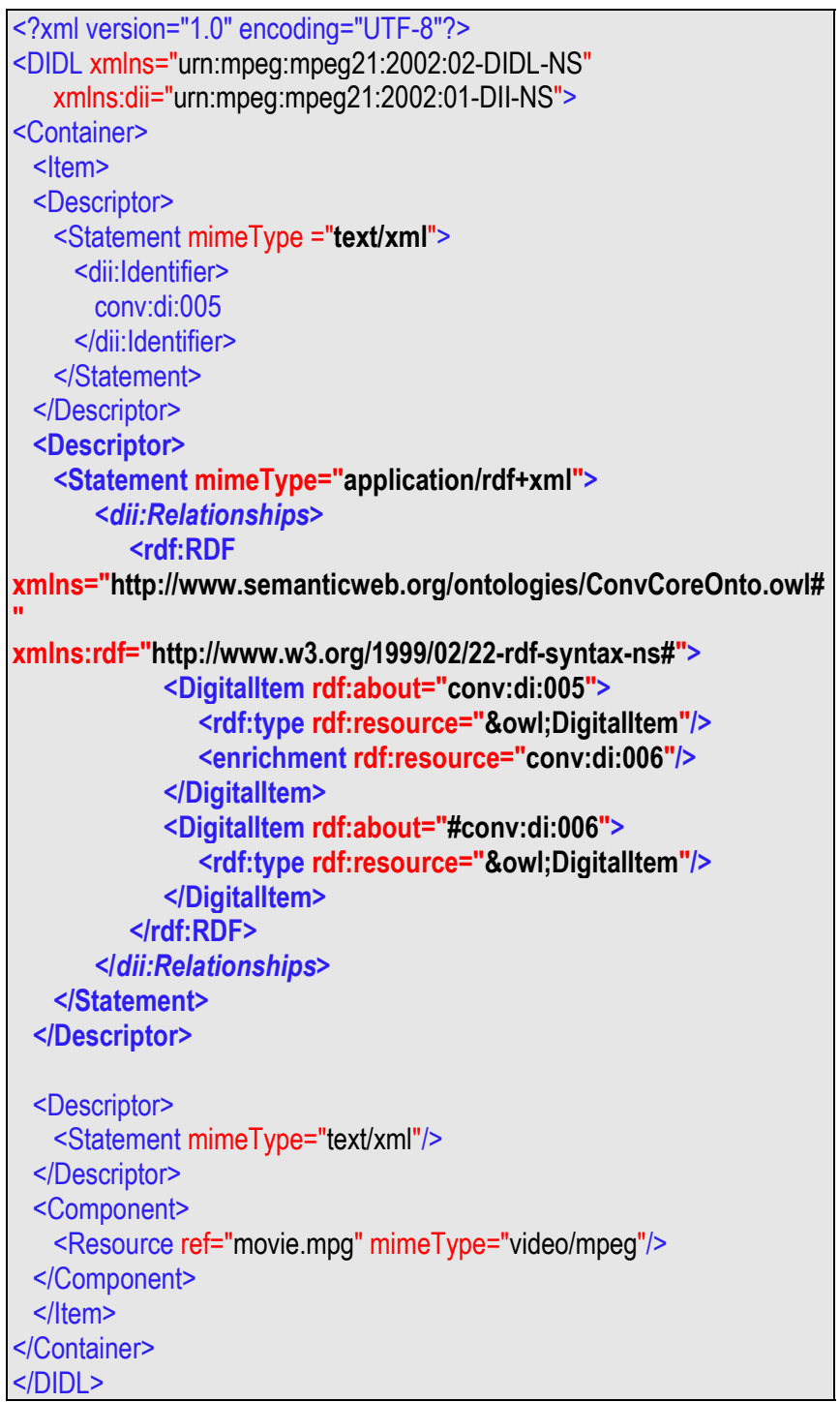

Figure 5 - Relationship Declaring MPEG-21 DI

\section{B. DI Search}

The existence of semantic information on inter-DI relationships may be exploited during search in various ways to enhance both search quality and user experience. On the one hand this information may be used to achieve high search recall and precision, the classic evaluation measures of search techniques from information retrieval science. On the other hand it may be provided as new ground to application developers to build or upgrade some functionalities to semantic functionalities, e.g. transforming simple keyword search into semantic search. In both cases the end user is the benefitted party. Possible exploitation mechanisms, which achieve the above goals, are presented in the following paragraphs.

Mechanisms presented here are based on the on-demand exploitation of the inter-DI relationships to provide richer search results, by reformulating the search query. The ondemand aspect is due to the fact that not all searches have the 
same requirements and should thus be handled differently [10]. In this context, inter-DI relationships are exploited in various ways, according to user search goals, to achieve better precision or recall. Precision and recall are in a fundamental tension. That is, a user performing an informational search wishes to have high recall over high precision so as to retrieve as much information as possible around a certain topic. But when a user searches for something specific he wishes high precision over high recall.

This way, high recall is achieved based on reasoning over inter-DI relationships that fall within a single ontology. InterDI properties may be connected with $r d f$ :subProperty $O f$ and search queries for DIs having a certain relationship with a DI may be expanded to include DIs that have a subProperty relationship with it, e.g. extension, completion, enrichment sub-properties.

But given that inter-DI relationships can be expressed using more than one ontology, high recall can also be achieved with reasoning over inter-DI properties of different ontologies. Properties that inter-connect through relationships like owl:sameAs or skos:narrower can also be used to expand the user query.

Additionally, in searches that have very low recall, an attempt at broadening the semantic target of the search query could be performed by using inter-DI properties that happen to be connected with the skos:broader property, trading this way precision for recall, basically following the opposite of the above strategies, i.e. going from a narrower concept to a broader one. Figure 6 presents an example of the mentioned procedure.

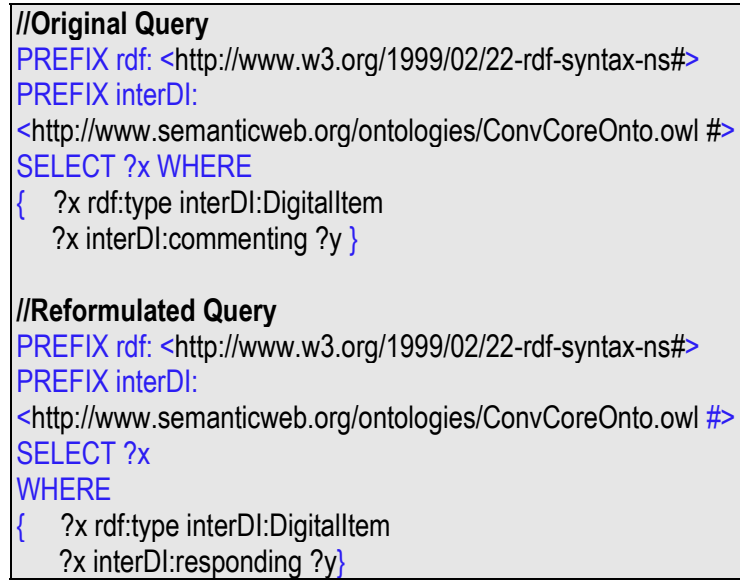

Regarding improved user experience, the use of the proposed mechanisms means that the user has the added value of understanding details about how two or more DIs relate to each other and the possibility to choose results that best fit his criteria. Furthermore, it also enables the evolution of applications from standard to semantic aware, giving developers a field to create more engaging user interfaces and functionalities, such as DI semantic browsing, faceted search or search via graphical inter-DI ontology traversing.

\section{CONCLUSIONS}

The growing complexity and interconnectivity of today's real and virtual worlds imposes an increasing need for more coherent and universal ways of declaring digital objects and for more precise and semantically enabled ways to specify the relationships between such resources.

MPEG standards are a step forward in the right direction. However, (as it was verified during the course of CONVERGENCE's use cases specification work), they still present some of the same shortcomings as those observed in today's predominant data declaration and structuring technologies, when they face inter-relating of digital items.

The work summarized in this paper, presents an extension to the MPEG-21 DII standard that, in a simple and efficient manner, greatly empowers it to perform a semantically precise declaration of DI relationships. The presented exploitations of the specified mechanism, within the context of CONVERGENCE, demonstrate its validity and universality for the declaration of relationships between digital resources, and show the added value of its widespread employment.

There is still ample room for development in the field of inter-resource relationship declaration. Future developments may focus, for instance, on the conception of new types of automatic exploitation of inter-DI relating metadata, and on the advancement and enrichment of the relationship declaration technologies/languages.

\section{ACKNOWLEDGMENT}

The authors would like to thank all the CONVERGENCE partners for the fruitful collaboration, in particular those who have contributed to the development of the CONVERGENCE use cases.

\section{REFERENCES}

[1] E. Aitenbichler, A. Behring, D. Bradley, T. Strufe, "Shaping the Future Internet”, Ubiquitous Computing and Communication Journal, Vol. 5(2), 2010.

[2] T. Zahariadis, P. Daras, J. Bouwen, N. Niebert, D. Griffin, F Alvarez, G. Camarillo, "Towards a Content-Centric Internet", In G. Tselentis et al. (Eds.) Towards the Future Internet - A European Research Perspective, IOS Press, 2010

[3] HTML Working Group, "W3C HTML 4.01 Specification, Section 12-Links", W3C, 1997 , http://www.w3.org/TR/html401/struct/links.html

[4] CONVERGENCE, CONVERGENCE project home site, 2011, http://www.ict-convergence.eu

[5] I. Burnett, S. Davis, G. Drury, "MPEG-21 Digital Item Declaration and Identification - Principles and Compression", IEEE Transactions on Multimedia, Vol. 7(3), 2005.

[6] MPEG-21, "ISO/IEC FDIS 21000-2:2005(E) MPEG-21 - Part 2: Digital Item Declaration", ISO, 2005.

[7] MPEG-21, "ISO/IEC 21000-3:2003/FDAM 1:2006(E) MPEG-21 Part 3: Digital Item Identification, AMENDMENT 1: Relates identifier types", ISO, 2006.

[8] MPEG-21, "ISO/IEC FDIS 21000-3 MPEG-21 - Part 3: Digital Item Identification", ISO, 2002.

[9] V. Jacobson, D. K. Smetters, J. D. Thornton et al., "Networking named content", ACM CoNEXT 2009

[10] A. Broder,"A taxonomy of web search". SIGIR Forum 36, 2 (September 2002), 3-10, 2002. 